\section{Großes Spektrum für die differenzierte Therapie}

Für die Behandlung der CED steht mittlerweile eine Vielzahl von Optionen zur Verfügung, die situationsgerecht eingesetzt werden sollten. Dr. Stephan Buderus, Bonn, betonte, dass für bestimmte Probiotika (E. coli Nissle oder VSL\#3 ${ }^{\oplus}$ ) die Wirksamkeit belegt werden konnte - insbesondere für die Pouchitis bei kolektomierten Patienten mit Colitis ulcerosa (Abb.). Spezialnahrungen können beim M. Crohn vergleichbar effektiv sein wie eine Steroidtherapie. Dabei müssen sich jedoch die Patienten in der Regel für sechs Wochen exklusiv von diesen therapeutischen Formula ernähren, nur Wasser und zuckerfreier Kaugummi sind zusätzlich erlaubt.

5-Aminosalicylate wie Mesalazin haben sich bei leichter bis mittlerer Entzün-

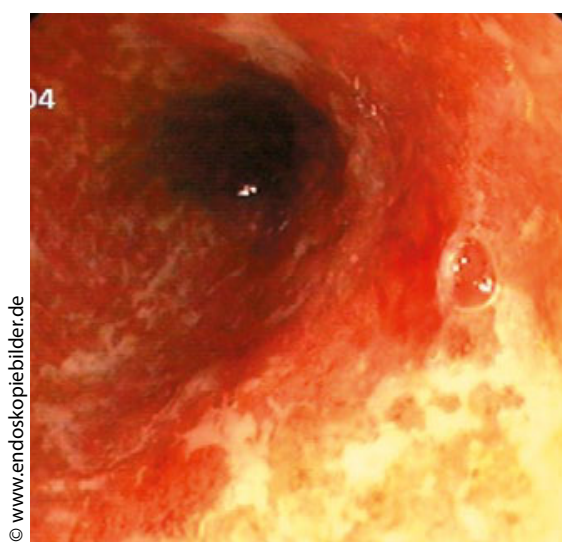

Abb.: Colitis ulcerosa

dungsaktivität bewährt. Sie werden auch zur Erhaltungstherapie insbesondere bei der Colitis ulcerosa häufig gegeben. Pred- nisolon wird bei mittlerer und hoher Entzündungsaktivität eingesetzt. Budesonid mit hoher Bindung an den Steroidrezeptor zeigt ein günstigeres Nebenwirkungsprofil, es wird in Kapselform bzw. Granulat zur lokalen Therapie des ileozökalen M. Crohn gegeben. Auch für die rektale Applikation stehen Budesonid -Präparate zur Verfügung.

Häufig ist zur Erhaltungstherapie ein Immunsuppressivum notwendig; in der Regel wird dazu Azathioprin eingesetzt. Inhibitoren des Tumornekrosefaktors alpha (TNF- $\alpha$ ) wie Infliximab haben ihren Schwerpunkt bei schwerem M. Crohn, der auf eine konventionelle intensive Therapie nicht anspricht. Dr. Andreas Fischer

Buderus S. Therapie der chronischentzündlichen Darmerkrankungen (CED)

- von Probiotika bis Biologika

\section{Buchtipps}

\section{Unübersehbar}

„Vertraute Gegenstände wie Äpfel und Apfelsinen sahen plötzlich fremd aus, so unvertraut wie eine asiatische Frucht." Hier beschreibt nicht ein Mensch seine Drogenerlebnisse, sondern seine ihn völlig überraschende Wahrnehmung morgens nach dem Aufstehen - er hatte unbemerkt einen Infarkt im Bereich seiner Sehrinde erlitten. Wie wichtig unsere visuelle Wahrnehmung, Speicherung und Verarbeitung für den Alltag sind, führt uns der Autor in Form von Biographien seiner Patienten vor Augen. Das Buch ist spannend zu lesen und vermittelt eine neue und bewusstere Sicht auf die "visuelle Welt".

Lesen - wenn es endlich erlernt ist - stellt für uns einen selbstverständlichen Vorgang dar. Umschriebene zerebrale Ausfälle im Bereich der okzipitalen Sehrinde, aber auch degenerative Prozesse zeigen, dass eine Vielzahl von Netzwerken an diesem Vorgang beteiligt ist. Die als Agnosien bezeichneten zentralen Sehstörungen umfassen die Prosopagnosie (Unfähigkeit, Gesichter zu unterschieden), räumlich visuelle Störungen bis hin zur Agraphie und Alexie.

In Form von Fallgeschichten erfährt der Leser nicht nur das medizinische Ausmaß verschiedener Störungen, sondern auch das „Erleben der Störung und das Leben mit der Störung". Eine der Fallgeschichten handelt vom Autor selbst: 2005 erkrankte Sacks an einem Optikusmelanom, das zu einem weitgehenden Sehverlust des rechten Auges führte. Der Wechsel von der Arzt- zur Patientenperspektive, gequält durch medizinisches Wissen, wird von ihm authentisch beschrieben. Kenntnisse darüber helfen im empathischen Umgang mit den eigenen Patienten.

Dr. Kirsten Stollhoff

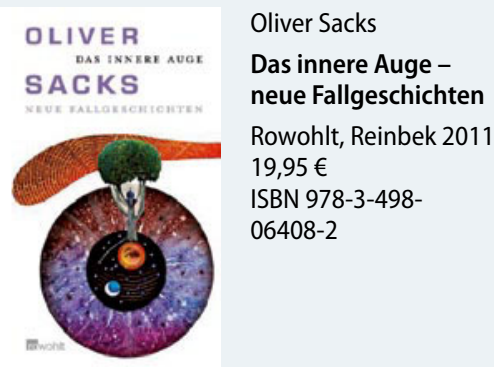

\section{Klassiker der Kinderdermatologie}

Die nach vier Jahren wieder neu überarbeitete und aktualisierte Auflage bietet zum gleichen Preis mehr als je zuvor: 77 Seiten, 120 Abbildungen und 30 Tabellen sind gegenüber der schon opulent ausgestatteten 2. Auflage von 2007 dazugekommen. Neue Kapitel umfassen Stoffwechselerkrankungen mit Hautbeteiligung, kutane Manifestationszeichen von
Immundefekten sowie die Hautpflege bei Neugeborenen und Säuglingen.

Peter Höger, Facharzt für Pädiatrie und Dermatologie, verhilft niedergelassenen wie klinisch tätigen Haut- und Kinderärzten mit diesem Buch, Erkrankungen der Haut bei Kindern und Jugendlichen besser zu erkennen und optimal zu behandeln. In seinem umfassenden Lehrund Nachschlagewerk werden alle häufigen und sehr viele seltene Dermatosen in ihrem komplexen Umfeld dargestellt. Alle Therapievorschläge sind hoch aktuell, die ausführlichen Tabellen zu Differenzialdiagnosen und Rezepturen vertiefen den flüssig geschriebenen Text. Zahlreiche Flussdiagramme helfen vom Symptom auf die richtige Diagnose zu schließen. Wie in seinen zahlreichen Vorträgen und Seminaren zeigt der Verfasser sein bewundernswertes Talent, den Leser nicht nur gut zu informieren, sondern ihn auch zu fesseln und v.a. für die Kinderdermatologie zu begeistern.

Dr. Ulrich Mutschler

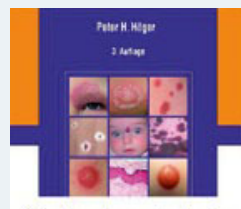

Peter Höger Kinderdermatologie Schattauer Verlag, Stuttgart 2011 $129,00 €$ ISBN 978-3-7945Kinderdermatologie 2730-4 